\title{
A hereditary angioedema screening in two villages, based on an index case, and identification of a novel mutation, "1033G $>T$ ", at the SERPING1 gene
}

\author{
Mehmet Yasar Ozkars¹, Ozlem Keskın², Nazan Bayram³, Huseyin Onay, Mehmet Keskın5, Hasan Bayram³, \\ Yavuz Sahın ${ }^{6}$, Ercan Küçükosmanoğlu², Serkan Kırık ${ }^{7}$

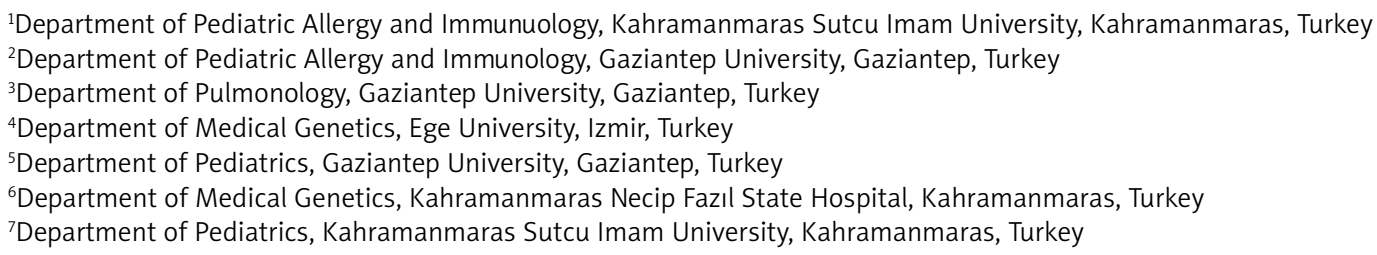

Adv Dermatol Allergol 2019; XXXVI (4): 403-411

DOI: https://doi.org/10.5114/ada.2018.78898

\begin{abstract}
Introduction: Hereditary angioedema (HAE) may be fatal and diagnosis can be delayed up to 10 years. We aimed to screen $\mathrm{HAE}$ in two villages based on an index case of HAE and to investigate for the mutation of the $\mathrm{C} 1$ esterase inhibitor (C1-INH) gene.

Material and methods: A total of 124 people were screened in two villages. The frequency and severity of symptoms were scored. C4, C1-INH levels and C1-INH activity were measured. We investigated for mutations of the C1-INH gene.

Results: Thirty-five cases of type I HAE and 7 cases of type II HAE were determined. Thirty-one (73.8\%) patients diagnosed with HAE were 18 years old or younger. There was a positive correlation between C4 levels, C1-INH levels $(p<0.0001, r=0.81)$, and C1-INH activity levels $(p<0.0001, r=0.631)$ and between the age at diagnosis and severity score $(p<0.0001, r=0.651)$. A positive correlation was found between the age at first symptom onset and C4 levels $(p=0.002, r=0.774)$, and C1-INH levels $(p=0.006, r=0.714)$. A marginally significant negative correlation was found between C1-INH activity levels and severity scores ( $p=0.1, r=-0.515)$. We identified a novel heterozygous 1033G > T missense variant of the C1-INH gene, SERPING1, in patients with type I HAE.

Conclusions: There are long delay periods in the diagnosis of HAE and when the index case is present, family screening may be very important and even life-saving, in particular, in paediatric patients without symptoms. Furthermore, the present study provides evidence to link a novel mutation, c.1033G $>$ T, to the development of HAE in a large HAE family from Turkey.
\end{abstract}

Key words: hereditary angioedema, SERPING1, 1033G>T.

\section{Introduction}

Hereditary angioedema (HAE) is a hereditary disease presenting with recurrent angioedema attacks. This swelling with unclear margins not accompanied by pain develops as a result of plasma postcapillary venules leaking in dermal layers of the dermis. This leakage basically derives from a lack of or insufficient activity of a protein called C1 esterase inhibitor [1, 2].
The disease is relatively rare. Reported frequencies range from $1: 10,000$ to $1: 150,000$ with $1: 50,000$ as the best overall estimate. This would indicate that there are roughly 500-7,000 people with HAE in Turkey [3-6].

This disease has hereditary properties and is considered autosomal dominant [7]. This means that it does not omit a generation and arise in each generation. No ethnic or sexual differences are known. C1 esterase inhibitor lev-

Address for correspondence: Serkan Kırık, Department of Pediatrics, Kahramanmaras Sutcu Imam University, 46100 Kahramanmaras, Turkey, phone: +90 5055771480, e-mail: srknkrk@hotmail.com Received: 30.11.2017, accepted: 1.05.2018. 
els are about 50\% in a heterozygote individual at birth, and symptoms do not generally start before $\mathrm{C} 1$ esterase inhibitor levels decrease below 35\%. Reduced C1 esterase inhibitor levels and the subsequent clinical presentation of HAE typically occurs within the first two decades [8]. The disease tends to deteriorate by puberty and then last for the remainder of individuals' lifetime.

The frequency and severity of attacks may vary greatly across patients and even in the same patient in different years [9]. The symptoms usually tend to be more severe in patients with early onset than in patients with late onset [10]. Some patients may experience mild attacks that recover without requiring any therapy, while others frequently have severe attacks that require emergency care. Moreover, the course of an attack cannot be estimated on the basis of prior baseline symptoms. Thus, the diagnosis of patients with discountable mild attacks, as early as possible, may prevent these patients from dying as a result of a laryngeal oedema attack.

Angioedema attacks are not often life-threatening, although they tend to be disruptive. An exception to this is the occurrence of swelling in the respiratory tract. More than $50 \%$ of cases of HAE experience at least one laryngeal oedema episode. Laryngeal symptoms generally appear at later ages and are rare before the age of 3 [11]. If the attack begins in the mouth and affects the respiratory tract, a clinical presentation blinding the glottis and leading to mortality with asphyxia may develop. The mortality rate may increase up to $50 \%$ in cases of undiagnosed HAE [12]. Although it has such a high mortality risk, HAE is diagnosed on average 8.5 years after the first symptoms appear [13]. It is essential to screen the relatives of index HAE patients for the disease.

C4 levels are low both during and between attacks for nearly all HAE cases. Thus, C4 is a very appropriate screening test in diagnosis. Subsequently, the determination of $\mathrm{C} 1$ esterase inhibitor levels and functioning helps both to diagnose and differentiate between type I (C1 esterase inhibitor level is low, C1 esterase inhibitor function is decreased) and type II HAE (C1 esterase inhibitor level is normal, C1 esterase inhibitor function is decreased) [14].

With HAE, one's mortality rate can rise up to $50 \%$ when the condition is left undiagnosed; thus, an early diagnosis and the use of medicines can save patients' lives.

\section{Aim}

We therefore aimed to screen all relatives of our patients who live in two different villages for HAE by looking at our type I HAE index case. We also investigated for mutations of the $\mathrm{C} 1$ esterase inhibitor gene.

\section{Material and methods}

\section{Subjects}

In our study, HAE screening was performed in two villages on a total of 124 individuals (in the first village
-70 , in the other village -54 ) as a result of the investigation conducted on relatives of the index case with a diagnosis of type I HAE. A family pedigree was constructed with 124 related individuals distributed in 3 generations. All individuals, with ages ranging from 1 to 82 years old, were interviewed and had blood samples collected and analysed for C4 and C1 esterase inhibitor levels. Physical examinations were performed. All 124 subjects were evaluated for HAE, including measurements of C4 and C1 esterase inhibitor and the diagnosis was established according to consensus criteria [11, 14]. C1 esterase inhibitor activity was investigated in patients with low C4 levels. We investigated for mutations of the C1 esterase inhibitor gene in 5 patients with $\mathrm{HAE}$ and 1 patient without HAE. Genomic DNA was extracted from peripheral blood. Complete sequencing of the coding region of the SERPING1 gene was carried out.

\section{HAE severity score}

A simplified HAE severity score was constructed based on the score proposed by the Third C1 Esterase Inhibitor Deficiency Workshop [15]. Episodes of angioedema were classified according to the average frequency and intensity of symptoms since the onset of the disease. The frequency of symptoms was quantitated as follows: more than 1 episode a month, 3 points; between 6 and 11 episodes a year, 2 points; $<6$ episodes a year, 1 point; and no symptoms of angioedema, 0 points. The intensity of symptoms was classified as follows: the presence of discomfort but no disruption in daily activity, 2 points; discomfort reducing normal daily activity, 4 points; and an inability to work or perform daily activity and/or the need for hospital care, 5 points. The sum of the frequency and intensity scores was used to classify the severity of the disease as follows: severe ( $\geq 7$ points); moderate (5 or 6 points); mild ( $\leq 4$ points); and asymptomatic (0 points).

\section{Measurement of $\mathrm{C} 1$ esterase inhibitor, $\mathrm{C} 1$ esterase inhibitor activity and C4 levels}

Serum C4 levels were determined by Siemens C4 Complement reagent, BN Prospec Nephelometry, with normal values ranging from 15 to $45 \mathrm{mg} / \mathrm{dl}$. Serum C1 esterase inhibitor levels were determined by Siemens C1 esterase inhibitor reagent, BN Prospec Nephelometry, with normal values ranging from 0.15 to $0.35 \mathrm{~g} / \mathrm{l}$.

Serum C1 esterase inhibitor activity levels were studied using TechnochromeC1 esterase inhibitor activity reagent, chromogenic (photometric) detection with Amax 200 analyser (C1 inhibitor functional levels $\leq 40 \%$ of normal are considered decreased).

\section{Identification of DNA mutations}

Selected by the geneticist, the gene assay was performed in 3 patients with type I HAE, 2 patients with type II, 
and 1 healthy person. Genomic DNA was extracted from peripheral blood. SERPING1 gene mutation analysis was performed by sequencing the coding exons and exon-intron boundaries of the gene in the Ege University, Department of Medical Genetics. Genomic DNA was extracted from peripheral EDTA anticoagulant whole blood using the MagNAPure LC automated system (Roche Applied Science, Manheim, Germany) following the manufacturer's instructions. To amplify the coding exons of the SERPING1 gene, primers were used, as listed in Table 1. The PCR fragments were sequenced by the Illumina MiSeq system using V2 chemistry (Illumina, Ca, USA). The sequencing results were analysed using IGV software (http://www.broadinstitute.org/igv/).

\section{Ethics approval}

All study procedures were done in accordance with a protocol previously approved by the Ethics Committee of the Gaziantep University. All parents provided written informed consent to the study procedures, and the children also gave consent.

\section{Statistical analysis}

A statistical software package was used for all data analyses and comparisons (SPSS v 11.5). The data were tested for assumptions of normalcy, and differences between the groups were compared with the Mann-Whitney $U$-test and $\chi^{2}$ test. A $p$-value of less than 0.05 was considered significant.

\section{Results}

The demographic characteristics of all screened individuals are shown in Table 2 and clinic and laboratory characteristics are shown in Table 3. The number of screened males was 60 (48.4\%) and females was 64 (51.6\%). Features of patients with HAE presented in Table 4.
Table 1. Primers used to amplify the coding exons of the SERPING1 gene

\begin{tabular}{ll}
\hline C1-INH 1-2F & TACAGGAACTCACACCAGCG \\
\hline C1-INH 1-2R & TTAATCCTCAGCCACCGCCC \\
\hline C1-INH 3F & TTTAGAGGACTGTGCCTCG \\
\hline C1-INH 3R & GGCTTTGTAAGTGTCTGG \\
\hline C1-INH 4F & CTCCAAAGCAGGGAATACC \\
\hline C1-INH 4R & GGTCTTCACCTGCTCTGCAG \\
\hline C1-INH 5-6F & CTCCACCATGCCGTATTCA \\
\hline C1-INH 5-6R & GGCTAGAGAAAGGAGAAGGAG \\
\hline C1-INH 7F & CAGACTGCAGGACAGCATTG \\
\hline C1-INH 7R & AACTTGCAGGGTTGCAGGAC \\
\hline C1-INH 8F & CTGGGACTCAGGATGAACCC \\
\hline C1-INH 8R & CAAGTCCAGGCAGGCAGCAG \\
\hline
\end{tabular}

\section{Results of C4, C1 esterase inhibitor and} C1 esterase inhibitor activity levels

C4 levels were low in 42 of the 124 screened individuals; 35 of these 42 individuals were diagnosed with type I $\mathrm{HAE}$ due to their low $\mathrm{C} 1$ esterase inhibitor levels and activity. C1 esterase inhibitor activity was also low in all patients with low C4 levels. Thus, 7 patients were diagnosed with type II HAE due to their low C4, low C1 esterase inhibitor activity and normal C1 esterase inhibitor levels. Forty-two individuals, or $30 \%$ of the screened individuals who were not diagnosed with HAE previously, were diagnosed with HAE by means of our screening, and patients were recommended appropriate therapies (C1 esterase inhibitor concentrate during HAE attack periods) after they were informed about the disease and given HAE patient cards.

The age at first symptom onset (mean: $12.38 \pm 10.49$ ) ranged between 1 and 40 years, while the age at diagnosis (mean: $16.95 \pm 14.82$ ) ranged between 1 and 58 years. Thirty-one patients (73.8\%) diagnosed with HAE were

Table 2. Demographic characteristics of all screened individuals

\begin{tabular}{|c|c|c|c|}
\hline Parameter & $\begin{array}{l}\text { Patients with } \\
\text { HAE }(n=42)\end{array}$ & $\begin{array}{l}\text { Healthy } \\
(n=82)\end{array}$ & $P$-value \\
\hline Age* $^{\star}$ & $16.95 \pm 14.82$ & $23.0 \pm 19.01$ & $0.14^{\#}$ \\
\hline Gender (M/F) & $24 / 18$ & $36 / 46$ & $0.165^{\star \star}$ \\
\hline C4 level $[\mathrm{mg} / \mathrm{dl}]^{*}$ & $7.08 \pm 2.64$ & $24.76 \pm 8.15$ & $<0.0001^{\#}$ \\
\hline $\mathrm{C} 1$ esterase inhibitor level $[\mathrm{g} / \mathrm{l}]^{\star}$ & $0.09 \pm 0.06$ & $0.27 \pm 0.05$ & $<0.001^{\#}$ \\
\hline $\mathrm{C} 1$ esterase inhibitor activity level (\%)* & $2.76 \pm 7.56$ & - & - \\
\hline HAE Severity Score* & $2.02 \pm 3.22$ & - & - \\
\hline Age $>18$ HAE Severity Score* & $5.36 \pm 3.52$ & - & - \\
\hline Age $\leq 18$ HAE Severity Score* & $0.84 \pm 2.11$ & - & - \\
\hline Age of the onset of the first symptoms* & $12.38 \pm 10.49$ & - & - \\
\hline Age of diagnosis* & $16.95 \pm 14.82$ & - & - \\
\hline
\end{tabular}


Table 3. Clinic and laboratory characteristics of healthy individuals and patients

\begin{tabular}{|c|c|c|c|}
\hline Parameter & Patients with HAE $(n=42)$ & Healthy $(n=82)$ & $P$-value ${ }^{\#}$ \\
\hline Male/female & $\begin{array}{c}\text { 24/18 (57/43\%) } \\
\text { 20/15 type I HAE } \\
\text { 4/3 type II HAE }\end{array}$ & $36 / 46(43.9 / 56.1 \%)$ & \\
\hline Symptoms of painful swelling in the body & $13(30.9 \%)$ & $23(28 \%)$ & 0.737 \\
\hline Symptoms of painful swelling on the face & $13(30.9 \%)$ & $23(28 \%)$ & 0.737 \\
\hline Symptoms of painful swelling due to trauma & $13(30.9 \%)$ & $23(28 \%)$ & 0.737 \\
\hline Symptoms of painful swelling after tooth extraction & $9(21.4 \%)$ & $6(7.3 \%)$ & 0.023 \\
\hline Symptoms of painful swelling after exercise, sorrow or alcohol intake & $13(0.9(\% 3)$ & $20(24.4 \%)$ & 0.436 \\
\hline $\begin{array}{l}\text { Symptoms of hoarseness, feeling of obstruction in the throat and } \\
\text { shortness of breath }\end{array}$ & $13(30.9 \%)$ & $18(22)$ & 0.275 \\
\hline Symptoms of severe abdominal pain & $13(30.9 \%)$ & $19(23.2 \%)$ & 0.351 \\
\hline $\begin{array}{l}\text { Symptoms of severe abdominal pain accompanied by vomiting and } \\
\text { diarrhoea }\end{array}$ & $13(30.9 \%)$ & $19(23.2 \%)$ & 0.351 \\
\hline $\begin{array}{l}\text { Symptoms of patients had undergone abdominal operations at any } \\
\text { time in their lives }\end{array}$ & $0(0 \%)$ & $0(0 \%)$ & 1.000 \\
\hline $\begin{array}{l}\text { Patients who were hospitalized in intensive care due to laryngeal } \\
\text { oedema }\end{array}$ & $1(2.3 \%)$ & $1(1.2 \%)$ & 0.628 \\
\hline Patients with low C4 level & $42(100 \%)$ & $0(0 \%)$ & $<0.001$ \\
\hline Patients with low $\mathrm{C} 1$ esterase inhibitor level & $35(83 \%)$ & $0(0 \%)$ & $<0.001$ \\
\hline Patients with low $\mathrm{C} 1$ esterase inhibitor activity level & $42(100 \%)$ & - & - \\
\hline
\end{tabular}

"Mann-Whitney $U$ test.

18 or younger; 11 of them (26.2\%) were older than 18 . Twenty-six of the 31 patients who were 18 and younger were diagnosed with type I HAE; 5 of them were diagnosed with type II HAE. Twenty-six of the 31 patients who were 18 and younger had no symptoms; 5 of them were symptomatic, and all of these patients with symptoms had a type I HAE diagnosis. We had 2 patients at the age of 1 and younger, and one of them was diagnosed with type I HAE and symptomatic at the same time; the other patient was diagnosed with type II HAE and asymptomatic. There were no age differences between diseased $(16.95 \pm 14.82)$ and healthy $(23.00 \pm 19.01)$ groups $(p=0.14)$.

The C4 and C1 esterase inhibitor levels of patients who were diagnosed with HAE were significantly lower than the healthy group $((7.08 \pm 2.64 \mathrm{mg} / \mathrm{dl}$ vs. $24.76 \pm 8.15$ $\mathrm{mg} / \mathrm{dl}, p<0.0001)$ and $(0.09 \pm 0.06 \mathrm{~g} / \mathrm{l} \mathrm{vs} .0 .27 \pm 0.05 \mathrm{~g} / \mathrm{l}$, $p<0.001$ ), respectively). A positive correlation was found between $\mathrm{C} 1$ esterase inhibitor and C4 levels in the entire group ( $p<0.0001, r=0.801, n=124$ ) (Figure 1 ). The mean C1 esterase inhibitor activity level of the diseased group was $2.76 \pm 7.56$. A positive correlation was found between the $\mathrm{C} 1$ esterase inhibitor activity levels and C4 levels of patients with HAE $(p<0.0001, r=0.631, n=42)$.

\section{Relationship between HAE severity scores and the age of patients with an HAE diagnosis}

Twelve of 35 patients (34.3\%) with type I HAE were symptomatic, while 1 of 7 patients (14.3\%) with type II
HAE was symptomatic. The HAE severity scores of patients with symptomatic type I HAE ranged between 2 and 5 (mean: $4.0 \pm 1.3,4.5$, IQR: 2.5-5), while the score of one symptomatic type II HAE patient was 4.

The severity scores of HAE patients who were 18 and younger (mean: $0.84 \pm 2.11)$ were lower than those of patients older than 18 (mean: $5.36 \pm 3.52)(p<0.0001)$. A positive correlation was found between the age at diagnosis and the severity score of patients with $\operatorname{HAE}(p<$ $0.0001, r=0.651, n=42$ ), while no correlation was found between the age at diagnosis and C4, C1 esterase inhibitor, and $\mathrm{C} 1$ esterase inhibitor activity levels. A positive correlation was found between the age of first symptom onset of symptomatic HAE patients and the age at diagnosis ( $p=0.006, r=0.714, n=13)$, C4 levels ( $p=0.001$, $r=0.824, n=13)$, and C1 esterase inhibitor activity levels ( $p=0.002, r=0.774, n=13)$; however, no correlation was found for $\mathrm{Cl}$ esterase inhibitor activity levels.

\section{Relationship between HAE severity scores and C1 esterase inhibitor activity levels}

The $\mathrm{C} 1$ esterase inhibitor activity levels of HAE patients with severity scores of 4 to 8 were significantly lower than those with severity scores of 0 to 3 ((0.01 (IQR 0.0-0.18) vs. $0.25(0.0-1.9) ; p=0.077)$ ) (Figure 2). While marginally significant, a negative correlation was found between C1 esterase inhibitor activity levels and severity scores in the HAE group older than 18 ( $p=0.1, r=-0.515, n=11)$; no 
Table 4. Features of patients with HAE

\begin{tabular}{|c|c|c|c|c|c|c|c|c|c|}
\hline No. & $\begin{array}{l}\text { Age } \\
\text { [years] }\end{array}$ & $\begin{array}{l}\text { Gender } \\
(M / F)\end{array}$ & $\begin{array}{l}\text { Age at onset of } \\
\text { symptoms [year] }\end{array}$ & $\begin{array}{l}\text { Age of diagnosis } \\
\text { [year] }\end{array}$ & $\begin{array}{l}\text { Severity } \\
\text { score }\end{array}$ & C4 [mg/dl] & $\begin{array}{c}\text { C1 esterase } \\
\text { inhibitor }[\mathrm{g} / \mathrm{l}]\end{array}$ & $\begin{array}{l}\mathrm{C} 1 \text { esterase inhibitor } \\
\text { activity (\%) }\end{array}$ & $\begin{array}{c}\text { HAE type } \\
\text { I/II }\end{array}$ \\
\hline 1 & 2 & $\mathrm{~F}$ & - & 2 & 0 & 7.39 & 0.11 & 2.9 & 1 \\
\hline 2 & 27 & $\mathrm{~F}$ & 10 & 27 & 8 & 5.6 & 0.06 & 0.03 & I \\
\hline 3 & 48 & $\mathrm{~F}$ & 15 & 48 & 8 & 5.6 & 0.08 & 0 & I \\
\hline 4 & 16 & $M$ & - & 16 & 0 & 5.6 & 0.03 & 0.25 & I \\
\hline 5 & 14 & $\mathrm{~F}$ & - & 14 & 0 & 5.6 & 0.06 & 0.14 & I \\
\hline 6 & 11 & $\mathrm{~F}$ & - & 11 & 0 & 5.8 & 0.08 & 0 & I \\
\hline 7 & 8 & $M$ & - & 8 & 0 & 5.6 & 0.06 & 0.37 & I \\
\hline 8 & 6 & $\mathrm{~F}$ & - & 6 & 0 & 5.6 & 0.09 & 0.9 & I \\
\hline 9 & 4 & $\mathrm{~F}$ & - & 4 & 0 & 5.6 & 0.08 & 1 & 1 \\
\hline 10 & 10 & $M$ & - & 10 & 0 & 5.63 & 0.07 & 0 & I \\
\hline 11 & 16 & $\mathrm{~F}$ & - & 16 & 0 & 5.6 & 0.04 & 0 & I \\
\hline 12 & 12 & $M$ & - & 12 & 0 & 5.6 & 0.05 & 0 & I \\
\hline 13 & 6 & $M$ & - & 6 & 0 & 5.6 & 0.04 & 0 & I \\
\hline 14 & 17 & $M$ & 5 & 17 & 8 & 5.6 & 0.04 & 1.2 & I \\
\hline 15 & 45 & $M$ & 15 & 45 & 6 & 5.6 & 0.08 & 0.14 & I \\
\hline 16 & 9 & $\mathrm{~F}$ & 2 & 9 & 5 & 5.6 & 0.09 & 0.2 & I \\
\hline 17 & 2 & $M$ & - & 2 & 0 & 6.33 & 0.14 & 0.09 & I \\
\hline 18 & 15 & $M$ & - & 15 & 0 & 5.6 & 0.08 & 0.25 & I \\
\hline 19 & 7 & $\mathrm{~F}$ & - & 7 & 0 & 7.58 & 0.12 & 0 & I \\
\hline 20 & 17 & $M$ & - & 17 & 0 & 5.6 & 0.08 & 0.03 & I \\
\hline 21 & 41 & $\mathrm{~F}$ & 15 & 41 & 8 & 5.6 & 0.07 & 0 & I \\
\hline 22 & 16 & $M$ & 5 & 16 & 7 & 5.6 & 0.04 & 0.14 & I \\
\hline 23 & 18 & $M$ & - & 18 & 0 & 5.6 & 0.03 & 0 & I \\
\hline 24 & 10 & $M$ & - & 10 & 0 & 5.6 & 0.04 & 1.07 & I \\
\hline 25 & 8 & $M$ & - & 8 & 0 & 9.95 & 0.08 & 0.14 & 1 \\
\hline 26 & 26 & $F$ & 15 & 26 & 8 & 5.6 & 0.05 & 0 & I \\
\hline 27 & 6 & $M$ & - & 6 & 0 & 5.6 & 0.09 & 0 & I \\
\hline 28 & 2 & $M$ & - & 2 & 0 & 6.71 & 0.11 & 2.19 & 1 \\
\hline 29 & 39 & $M$ & - & 39 & 0 & 5.6 & 0.06 & 0 & I \\
\hline 30 & 10 & $\mathrm{~F}$ & - & 10 & 0 & 12.8 & 0.26 & 38 & II \\
\hline 31 & 8 & $M$ & - & 8 & 0 & 11.3 & 0.2 & 9.7 & II \\
\hline 32 & 6 & $M$ & - & 6 & 0 & 5.6 & 0.07 & 1.24 & I \\
\hline 33 & 1 & $M$ & - & 1 & 0 & 5.6 & 0.16 & 6.1 & II \\
\hline 34 & 32 & $M$ & 16 & 32 & 7 & 5.6 & 0.05 & 0 & 1 \\
\hline 35 & 1 & $F$ & 1 & 1 & 3 & 5.83 & 0.08 & 0.71 & 1 \\
\hline 36 & 58 & $\mathrm{~F}$ & 20 & 58 & 8 & 12.2 & 0.07 & 0 & 1 \\
\hline 37 & 4 & $\mathrm{~F}$ & 2 & 4 & 3 & 5.6 & 0.07 & 0 & I \\
\hline 38 & 31 & $M$ & - & 31 & 0 & 9.07 & 0.11 & 0.48 & 1 \\
\hline 39 & 52 & $F$ & 40 & 52 & 6 & 13.5 & 0.2 & 0.31 & II \\
\hline 40 & 23 & $F$ & - & 23 & 0 & 14.1 & 0.24 & 24.2 & II \\
\hline 41 & 15 & $M$ & - & 15 & 0 & 11.4 & 0.21 & 21.9 & II \\
\hline 42 & 13 & $M$ & - & 13 & 0 & 12.1 & 0.22 & 2.25 & II \\
\hline
\end{tabular}



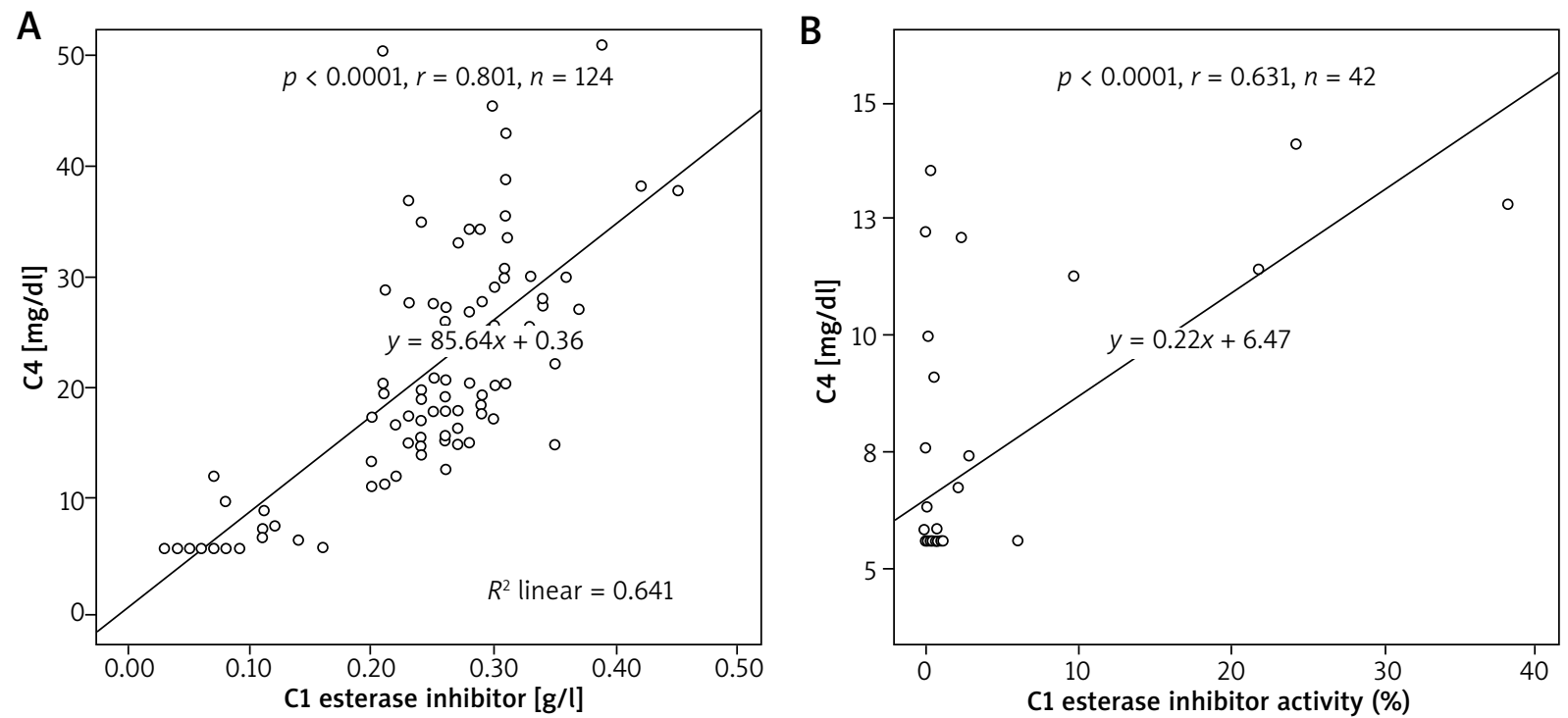

Figure 1. A - Correlation between C4 levels and C1 esterase inhibitor levels of cases. B - Correlation between C4 levels and $\mathrm{C} 1$ esterase inhibitor activity levels

correlation was found between C1 esterase inhibitor levels and severity scores $(p=0.14, r=-0.476, n=11)$.

\section{Mutation analysis of the SERPING1 gene}

We analysed three type I HAE patients, including our index case, and two type II HAE patients and one healthy person without HAE, for the mutation in the SERPING1 gene (Figure $3 \mathrm{~A}$ ). In the light of the clinical findings, the SERPING1 coding region (ENST00000278407) was analysed in our index case (III-3) and subjects II-15 and

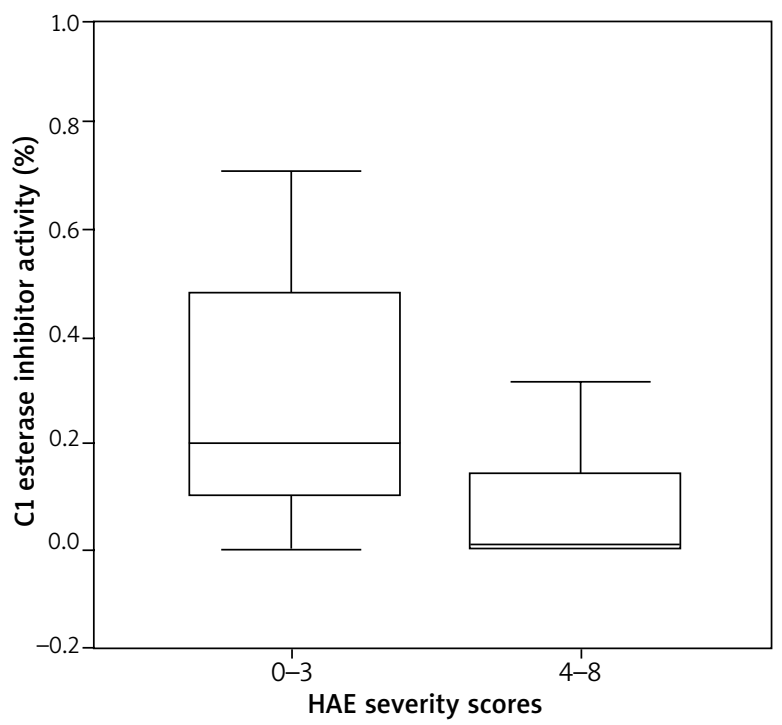

Figure 2. Relationship between HAE severity scores and C1 esterase inhibitor activity levels
III-11, and a novel heterozygous 1033G >T missense variant was identified in these patients with type I HAE, substituting tryptophan for the evolutionarily conserved glycine, located in the serpin protease inhibitor domain of the protein (p.Gly345Trp) (Figures 3 B, C). This variation is not reported in the dbSNP database (http://www. ncbi.nlm.nih.gov/SNP/), the 1000 genome database (www.1000genomes.org) and the human gene mutation database (http://www.hgmd.cf.ac.uk/ac/index.php). This mutation site is conserved among vertebrates and mutation prediction in silico programs, SIFT (http://sift.jcvi. org) and PolyPhen-2 (http://genetics.bwh.harvard.edu/ pph2/) predict that this mutation is significantly a disease causing one. In addition, to analyse the effect of this substitution on the protein structure, wild-type and the predicted p.Gly345Trp-SERPING1 protein sequences were evaluated using SWISS-MODEL (http://swissmodel.expasy.org) (Figure 3 D). The Z-score of the estimated model was -2.03 (PMID: 9504803, 16301204, 12824332). While all 3 patients with type I HAE were positive for the 1033G > T mutation, one healthy person without HAE, shown as II-12 in pedigree, had no defect in the SERPING1 gene. Finally, we could not detect any defect in the SERPING1 gene in 2 patients, represented as II-17 and IV-39 in pedigree, with type II HAE.

The family pedigree is shown in Figure $3 \mathrm{~A}$.

\section{Discussion}

In the present study, 124 patients from two villages were screened by looking at the index case in our study. Of them, 42 patients were diagnosed with HAE; 35 patients had type I HAE and 7 type II HAE, and $74 \%$ of these 
A

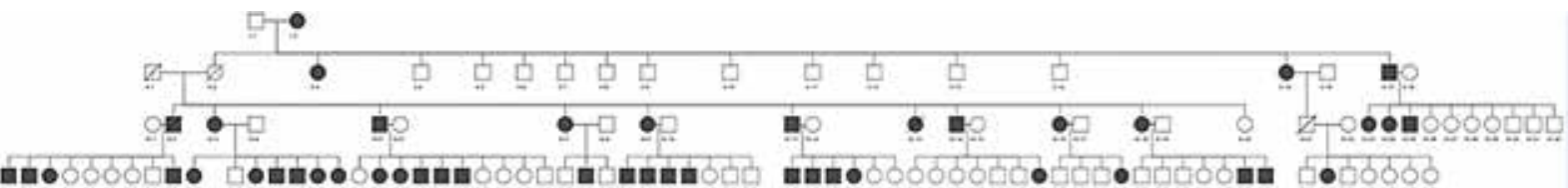

B

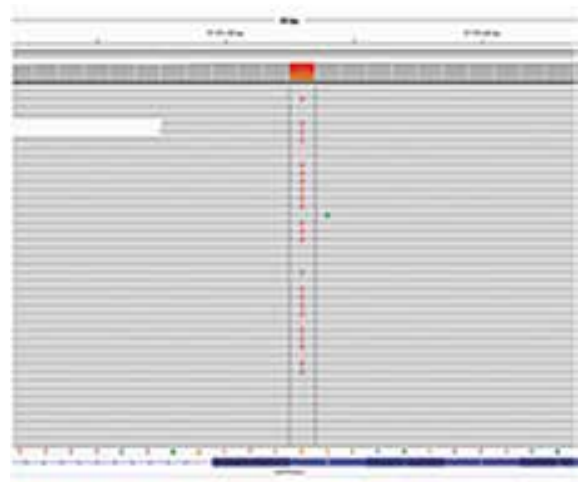

C

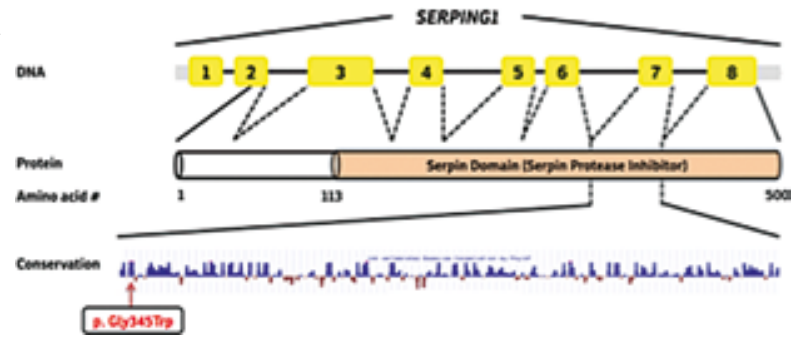

D

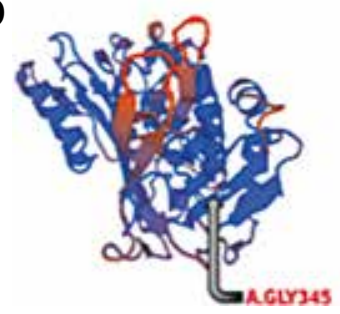

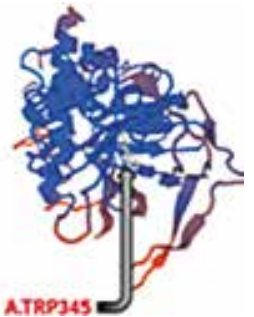

Figure 3. A - The HAE family pedigree. Note that the black boxes indicate type I HAE; however, subjects II.17, III.23, III.24, III.25, IV.38, IV.39 and IV.40 have type II HAE, interestingly, in the same family. B - Display of the Integrative Genomics Viewer (IGV) showing the SERPING1 novel heterozygous C.1033G>T missense variant. The grey letter represents the wildtype nucleotides and the red letter represents a single base-nucleotide alteration. C - The SERPING1 gene structure and the location of the functional domain (serpin protease inhibitor) are indicated. The conservation of $345^{\text {th }}$ glycine residue among species. Conserved glycine is shown with the arrow (Information Conservation). D - The wild-type protein and defective protein, respectively, covering the regions of serpin protease inhibitor, as presented in the SWISS-MODEL work space window

42 patients were 18 or younger. Approximately one out of every 3 patients was diagnosed with HAE. A positive correlation was detected between $\mathrm{C} 4$ levels and $\mathrm{C} 1$ esterase inhibitor levels and C1 esterase inhibitor activity levels and between the age at diagnosis and severity scores. A negative correlation was detected between $\mathrm{C} 1$ esterase inhibitor activity levels and severity scores. The C1 esterase inhibitor activity of patients with high HAE clinical severity was lower than those with low HAE clinical severity. We also reported an additional missense heterozygous c.1033G > T mutation (p.G345T) of the SERPING1 gene in patients with type I HAE.

Out of patients who could be diagnosed by means of our screening, 26 of 35 type I HAE patients and 5 of 7 type II HAE patients were 18 years old or younger. We consider that the quality of life and survival times of paediatric patients who have a long forthcoming lifetime would increase with early diagnosis.

Furthermore, 29\% of the 124 screened patients had an experience of body swelling, facial swelling and swelling as a result of trauma, and this was seen in $31 \%$ of patients in which HAE was detected. These results indi- cate that these questions are not helpful in this screening period.

Of the 124 patients screened, $12 \%$ experienced swelling due to tooth extraction. A total of $21 \%$ of the diseased group had swelling due to tooth extraction. The question about swelling following tooth extraction was observed to be a distinguishing question in HAE diagnosis.

In addition, 31\% of the diseased group experienced swelling at any time in their life after exercise or sorrow, $31 \%$ had a severe stomach ache at any time in their life, and in 31\%, the stomach ache was accompanied by vomiting and diarrhoea. None of the patients experienced abdominal surgery. These ratios are also similar to the ratio of $25 \%$ mentioned by Kasamatsu et al. [16]. A severe stomach ache was reported by Lei et al. in 18.2\% [17].

Moreover, $31 \%$ of the diseased group experienced hoarseness or an obstruction in their throat at any time in their life. Lei et al. [17] also found laryngeal oedema in $45.5 \%$. Three members of the family we screened had died due to laryngeal oedema and asphyxia; $2.35 \%$ of the diseased group received inpatient treatment in an intensive care unit at any time of their life. 
A total of $60 \%$ of screened individuals were 18 or younger. HAE was detected in $42 \%$ of 74 individuals in this age group. HAE was detected in $22 \%$ of 50 individuals who were older than 18 . None of the cases were previously diagnosed, except the index case. It should be noted that none of the patients who were diagnosed during our screening were previously diagnosed. This situation indicates the importance of screenings performed by looking at the index case. Patients do not suspect themselves to have the disease, although they have the same symptoms as their relatives who were diagnosed with HAE, and they try to keep the diagnosis away from them. Only $31 \%$ of all patients were symptomatic, compared to $57.9 \%$ of patients in the reports of Lei et al. [17] As most of the patients we diagnosed were younger than 18 , symptoms were considered as potentially not developed yet. Skin findings, laryngeal oedema findings and abdominal findings existed in the reports of all symptomatic patients. Skin findings were present in all symptomatic patients in a report by Lei et al. [17]. One unique family had 4 members who were diagnosed with type I or type II HAE in the same family. In this family, 3 siblings were diagnosed with type II, while 1 sibling was diagnosed with type I HAE.

Delay in diagnosis would likely have been much longer in some patients without screening. This delay was found to be 8.45 years in the study conducted by Lei et al. [17]. The average age at diagnosis was 16.95, with a diagnosis delay of 4.57 years in our study with the aid of our screening.

As well as having low C1 esterase inhibitor levels from birth, symptoms appear when C1 esterase inhibitor levels fall below $35 \%$ by the nature of the disease as the patient grows up and HAE symptom scores are increased. In support of this, in the HAE patients that we diagnosed, symptoms appeared as the patients grow up, and there was a negative correlation between symptom scores and C1 esterase inhibitor activity in patients older than 18 years. Furthermore, it was demonstrated that the $\mathrm{C} 1$ esterase inhibitor activity of patients with high HAE clinical severity was lower than those with low HAE clinical severity. Similarly, a relationship was discovered between $\mathrm{C} 1$ esterase inhibitor activity levels and HAE severity in a study conducted by Ferraro et al. [18]. In our study, we could detect marginally significant relationships between $\mathrm{Cl}$ esterase inhibitor activity and HAE symptom scores due to the fact that we had a few patients older than 18 and most of the patients under 18 were asymptomatic.

In our study, we found that symptom scores were high in HAE patients with lower diagnosis age. These results are in accordance with those reported in the literature [10]. In addition, we showed in our study that screening tests may provide better results than surveys as patients are often asymptomatic at early ages. Thus, screening tests should be performed on the relatives of the patients with HAE and early diagnosis should be achieved. Ferraro et al. emphasized that the initial symptoms may not appear until after 40 years of age; therefore, particularly during younger ages, a lack of symptoms does not exclude the possibility of the disease [18]. Hence, our patients who were older than 18 were detected to have higher HAE symptom scores than those who were under 18.

Another important finding we detected during our screening was the existence of a very strong correlation between $\mathrm{C} 4$ levels and $\mathrm{C} 1$ esterase inhibitor levels and activity. This result once more revealed that the use of a simple C4 test was an accurate and strong tool to be used for HAE screening.

Finally, as another important finding of our study, here we report an additional missense heterozygous c.1033G > T mutation (p.G345T) of the SERPING1 gene in patients with type I HAE. The missense variant found in the evolutionarily ultra-conserved serpin protease inhibitor domain of the C1-Inhibitor (C1Inh) gene and cause hereditary angioedema.

\section{Conclusions}

There are long periods of delay in the diagnosis of HAE and when the index case is present, family screening may be very important, and even life-saving. In particular, in paediatric patients without symptoms, screening tests are more important as surveys are inadequate in diagnosing. Furthermore, the present study provides evidence to link a novel mutation, c.1033G>T, to the development of HAE in a large HAE family from Turkey.

\section{Conflict of interest}

The authors declare no conflict of interest.

\section{References}

1. Temino VM, Peebles RS. The spectrum and treatment of angioedema. Am J Med 2008; 121: 282-6.

2. Bernstein IL. Hereditaryangioedema: a current state-ofthe-art review. II: historical perspective of non-histamineinduced angioedema. Ann Allergy Asthma Immunol 2008; 100: 2-6.

3. Nzeako UC, Frigas E, Tremaine WJ. Hereditary angioedema: a broad review for clinicians. Arch Intern Med 2001; 161: 2417-29.

4. Weldon D. Differential diagnosis of angioedema. Immunol Allergy Clin North Am 2006; 26: 603-13.

5. Kulthanan K, Jiamton S, Boochangkoot K, Jongjarearnprasert K. Angioedema: clinical and etiological aspects. Clin Dev Immunol 2007; 2007: 26438.

6. Zuraw BL. Clinical prectice. Hereditary angioedema. N Engl J Med 2008; 359: 1027-36.

7. Zuraw BL. Hereditary angioedema: a current state-of-the art review. IV: short- and long-term treatment of hereditary angioedema: out with the old and in with the new? Ann Allergy Asthma Immunol 2008; 100: 13-8. 
8. Bork K, Meng G, Staubach P, Hardt J. Hereditary angioedema: new findings concerning symptoms, affected organs, and course. Am J Med 2006; 119: 267-74.

9. Winnewisser J, Rossi M, Späth P, Burgi H. Type I hereditary angio-oedema: variability of clinical presentation and course within two large kind reds. J Intern Med 1997; 241: 39-46.

10. MacGinnitie AJ. Pediatric hereditary angioedema. Pediatr Allergy Immunol 2014; 25: 420-7.

11. Bowen T, Cicardi M, Bork K, et al. Hereditary angioedema: a current state-of-the-art review. VII: Canadian Hungarian 2007 International Consensus Algorithm for the diagnosis, therapy, and management of hereditary angioedema. Ann Allergy Asthma Immunol 2008; 100: 30-40.

12. Bork K, Hardt J, Witzke G. Fatal laryngeal attacks and mortality in hereditary angioedema due to C1-INH deficiency. J Allergy Clin Immunol 2012; 130: 692-7.

13. Zanichelli A, Magerl M, Longhurst H, et al. Hereditary angioedema with $\mathrm{C} 1$ inhibitor deficiency: delay in diagnosis in Europe. Allergy Asthma Clin Immunol 2013; 9: 29.

14. Tarzi MD, Hickey A, Föster T, et al. An evaluation of tests used for the diagnosis and monitoring of $\mathrm{C} 1$ inhibitor deficiency: normal serum C4 does not exclude hereditary angiooedema. Clin Exp Immunol 2007; 149: 513-6.

15. Agostoni A, Aygoren-Pursun E, Binkley KE, et al. Hereditary and acquired angioedema: problems and progress: proceedings of the third $\mathrm{C} 1$ esterase inhibitor deficiency workshop and beyond. J Allergy Clin Immunol 2004; 114: 51-131.

16. Kasamatsu Y, Yoshinoya K, Kasamatsu Y, et al. A case of hereditary angioedema involving recurrent abdominal attacks. Intern Med 2011; 50: 2911-4.

17. Lei WT, Shyur SD, Huang LH, et al. Type 1 hereditary angioedema in Taiwan-clinical, biolocical features and genetic study. Asian Pac J Allergy Immunol 2011; 29: 327-31.

18. Ferraro MF, Moreno AS, Castelli EC, et al. A single nucleotide deletion at the $\mathrm{C} 1$ inhibitor gene as the cause of hereditary angioedema: insights from a Brazilian family. Allergy 2011; 66: 1384-90. 